\title{
Study on Hydrodynamics of a New Comb-type Floating Breakwater Fixed on the Water Surface
}

\author{
Yaoyong Chen ${ }^{1, *}$, Guoxu Niu ${ }^{1}$, and Yuliang $\mathrm{Ma}^{2}$ \\ ${ }^{1}$ Wenzhou Vocational \& Technical College, China \\ ${ }^{2}$ Engineering College, Ocean University of China, China
}

\begin{abstract}
Through the physical model cross-section experiment, the effects of the relative width and groove depth on the transmission coefficient, horizontal wave force and vertical wave force of the new comb-type floating breakwater (FBW) model under fixed condition are observed. The results show that the hydrodynamic parameters of the new comb-type FBW are mainly influenced by its relative width under the action of regular wave, and the transmission coefficient decreases with the increase of its relative width. Especially when the relative width is 0.139 to 0.188 , the transmission coefficient of the new comb-type FBW decreases rapidly with the increase of the relative width, and the horizontal wave force and the vertical wave force change slowly. This indicates that the new comb-type FBW has obvious effect on wave dissipation about short and medium waves. In addition, numerical investigations of selected experiment cases are conducted using RANS based commercial CFD code Flow3D. The numerical results show a good ability to capture the hydrodynamic interaction effect of the fixed FBW.
\end{abstract}

\section{Introduction}

The FBW is an important port and coastal engineering building, shields the port and related waters mainly. Compared with the traditional bottom-type FBW, the new comb-type FBW not only reduce the wave energy, but also has the characteristics of low cost, convenient transportation, easy demolition, without damaging to the ecological environment of the water, and suitable for the soft soil seabed waters. At present, the FBWs are used to cover deep-water port and wharf waters, cover waters such as aquaculture and bathing beaches, cover offshore construction sites, cover the waters of military jets at sea, and a wave prevention measure for marine disaster prevention and emergency response (Wang, 2010). Therefore, more and more scholars pay attention to the FBWs. Moreover, the FBWs have certain engineering application examples at home and abroad.

Among many FBWs, rectangular box-type breakwater and based on the improvement of it have always been the focus of scholars at home and abroad. In addition, M. J. Alizadeh (2014) points out FBWs fixed in semi-submerged state are better than floating breakwaters with single degree of freedom (vertical direction) and moored state in the aspect of the wave energy dissipation characteristics. So it is very necessary to study the FBW under fixed in semi-submerged state. Based on the linear time-domain theory, Li Yucheng (2002) establishes the wave time-domain integral equation of the box-type FBW, and applies the boundary element method to solve its horizontal and vertical wave forces fixed on the water surface. Chen $\mathrm{Li}$ (2002) designs the square box-type FWB with the same width as the wave flume, and measures the wave height and the pressure on the surface of the FBW by using the waveheight gauge and pressure sensor, then studies the wave energy dissipation characteristics of the two-dimensional fixed FBW and the variation and distribution of the surface pressure. Zou Zhili (2003) measures the horizontal wave force and vertical wave force of the FBW model fixed on the water surface. It is pointed out that the horizontal wave force is proportional to the draft, the relative width and the wave height, and the vertical wave force was proportional to the relative width, the wave period and the wave height. However, it does not carry out systematic data analysis, but study the nonlinear interaction between diffracted wave and incident wave of FBW. In order to study the degree of freedom's influence on the hydrodynamic parameters of FBW, Alizadeh, M.J. (2014) carries out T-type FBW physical model experiments with fixed state and single degree of freedom (heave). It is found that the wave energy dissipation characteristics of T-type FBW with single degree of freedom (heave) is more obvious than that fixed on the water surface, but the wave reflection of T-type FBW with two arrangement forms is just the opposite under the same wave period. Beyond that, four different FBWs configurations are examined under the action of regular and irregular waves by E.V. Koutandos, P.E. Prinos (2005). It is mentioned that the reason of the energy dissipation is the structure has strong vibration, and air-water vortices occurs near the structure. Tanvir Sayeed (2017) reveals that the commercial CFD software Flow3D shows good agreement with the 
experimental data in investigating the change in wave loads for different sizes of small spherical bodies at different proximities to a fixed structure. Jun Jin (2011) uses software Flow-3D to analyze the effects of green water loading and superstructure elevation on wave forces.

Based on the previous studies, this paper refers to the experimental model of bottom-founded comb-type breakwater by Zhuo Fang (2011), the rectangular box FBW is reconstructed into a comb-type floating form. And all FBWs have been constructed out of solid structure. Owing to its cutting-edge contour of the reconstructed structure is not a straight line, the wave cannot act on the FBW at the same time, thus reducing the simultaneous action of waves on breakwater. The horizontal wave force also decreases accordingly. By changing the relative width of the FBW, the rules of transmission coefficient and wave force of the new comb-type FBW are discussed in this paper.

\section{Experimental investigation}

\subsection{Facility layout and model design}

In order to study the hydrodynamic characteristics of the FBW, all the experiments have been performed at the new wave flume in the Hydrodynamic Laboratory of Laoshan Campus, Ocean University of China. The wave flume is 25 meters long, 1 meter wide and 1.2 meters high, and the maximum water depth is $1 \mathrm{~m}$. One end of the flume is equipped with a wave generator and wave damping filling material is equipped in the other end of the flume, the experimental water depth is $0.74 \mathrm{~m}$. The model which has a uniform density is made of hardwood boards, has been placed in the center of the flume with a draft of $0.18 \mathrm{~m}$. The scale ratio is nominally $1: 40$. The wave forces acting on the floating body are measured by tri-direction-force transducer which is fixed on the flume. The size of the FBW and the arrangement of the experimental equipment in the wave flume have been shown in Figure 1 and Figure 2, a tri-direction-force transducer is installed directly above the gravity center of the FBW to measure the horizontal and vertical wave forces simultaneously. Because the wave force is tremendous when the wave acts on the FBW, the experimental model is fixed by a dedicated bracket in the flume, and its elevation can be adjusted according to the experiment requirements. The wave element is measured by capacitive wave-height gauge. Two wave-height gauges on the offshore side are measured the transmission wave height. The other two on the onshore side are measured the wave height in front of the FBW. Besides, the four wave heights also can be read by these two wave heights in the process of wave filtering, so as to correct the wave height and period that needed promptly. And all measuring instruments have been calibrated before and after the experiments. They are proved to be accurate.

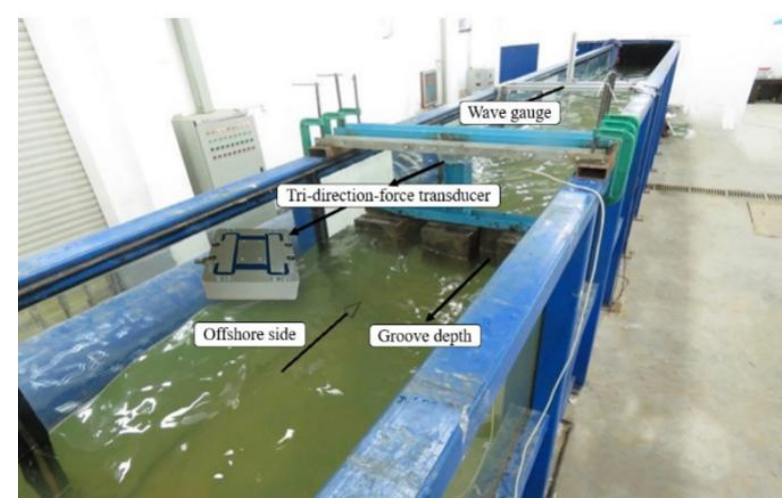

Figure 1 The picture of experimental model and equipment layout

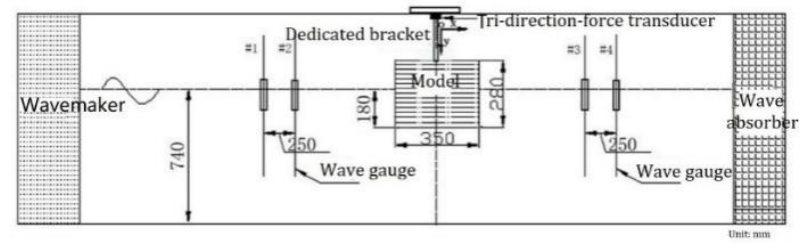

Figure 2 Schematic layout of the experimental set up in flume

To make it easier to describe the elements of the experiment, the symbols which are related to the experiment have been shown in Table 1. Eight regular waves with three different wave steepness were selected with wave lengths as multiples of the fixed structure diameter and also to cover full scale wave periods of 5.6-10 s. The wave particulars are given in Table 2 .

Table 1 Experimental related symbols

\begin{tabular}{cc}
\hline The main parameters & Symbol \\
\hline Incident wave height & $\mathrm{H}$ \\
Floating breakwater width & $\mathrm{B}$ \\
Wavelength & $\mathrm{L}$ \\
Groove depth & $\mathrm{Q}$ \\
Transmission coefficient & $\mathrm{Kt}$ \\
Wave period & $\mathrm{T}$ \\
Relative width & $\mathrm{B} / \mathrm{L}$ \\
Horizontal wave force & $\mathrm{Fx}$ \\
Vertical wave force & $\mathrm{Fz}$ \\
\hline
\end{tabular}

Table 2 Wave particulars

\begin{tabular}{cccc}
\hline Model name & $\begin{array}{c}\text { Wave } \\
\text { height }(\mathrm{m})\end{array}$ & $\begin{array}{c}\text { Groove } \\
\text { depth }(\mathrm{m})\end{array}$ & $\begin{array}{c}\text { Wave period } \\
(\mathrm{s})\end{array}$ \\
\hline $\begin{array}{c}\text { New comb- } \\
\text { type FBW }\end{array}$ & $\begin{array}{c}0.05 / 0.07 / \\
0.09\end{array}$ & $0 / 0.14 / 0.28$ & $0.9 / 1.0 / 1.1 / 1.2 /$ \\
\hline
\end{tabular}

\subsection{Data processing}

The time domain curves of the wave height data, the horizontal wave force and the vertical wave force of the new comb-type FBW need to be collected during the experiment simultaneously. The sampling time interval of wave-height gauge is $0.02 \mathrm{~s}$, the sampling interval of tri-direction-force transducer is $0.01 \mathrm{~s}$, and the sampling time of each experiment condition is $40 \mathrm{~s}$. The wave height is measured by a wave-height gauge while the wave force is read by a tri-direction-force transducer with a measuring range of $( \pm 5000 \mathrm{~N})$. In terms of numerical choice, the mean values of peak to peak of the 
time-history curve are calculated as the transmission wave height, horizontal and vertical wave forces of the FBW under a certain experiment condition. Figures 3, 4 and 5 show the wave surface variation, horizontal and vertical wave forces time domain curves of the FBW at 1.1 second wave period. The wave force of the collection has been drew into a time domain analysis curve, which is shown in Figure 4, 5. The average values of peak to peak value of wave force time domain curve are plotted in Figures 7, 8, 10, 11.

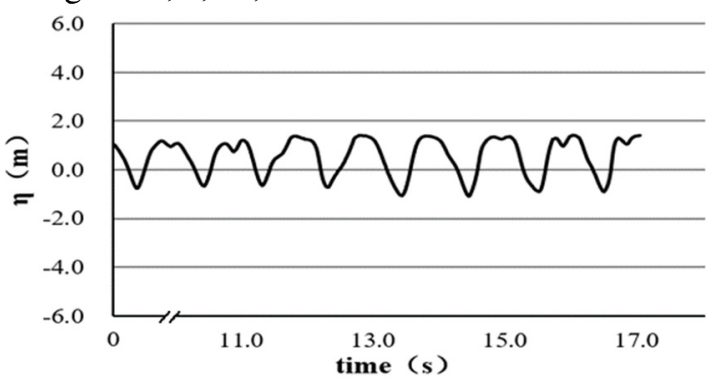

Figure 3 The time domain curve of wave variation

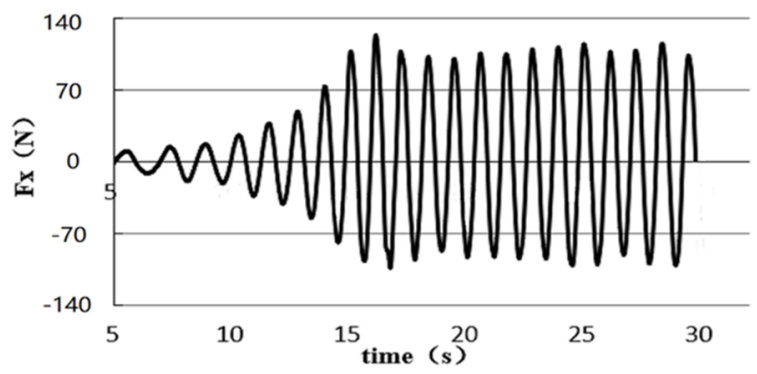

Figure 4 The time domain curve of horizontal wave force

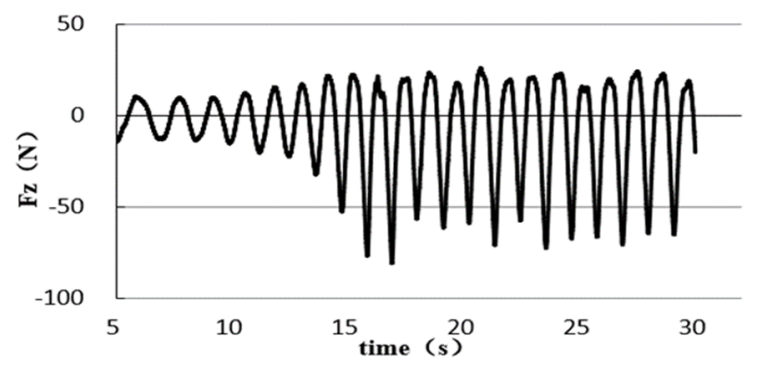

Figure 5 The time domain curve of vertical wave force

As can be seen from Figure 4, the waveform is a bit irregular. Because there are vortexes on the front and back of the FBW when wave act around it. The position changes of the vortexes make the wave surface behind the FBW irregular. Fortunately, it has little effect on the wave height. The coordinate axis of the tri-directionforce transducer can be seen in Figure 2. We can see that the horizontal wave force on the offshore side is greater than that on the onshore side from Figure 5, which is caused by the instantaneous impact of waves on the FBW. Figure 6 reveals the vertical upward wave forces are greater than that vertical downward wave forces, because the wave height above the wave surface is larger than that below the water surface in the Stokes wave.

\section{Experimental results and discussion}

\subsection{Effect of groove depth and wave height on hydrodynamic characteristics of the new comb- type FBW}

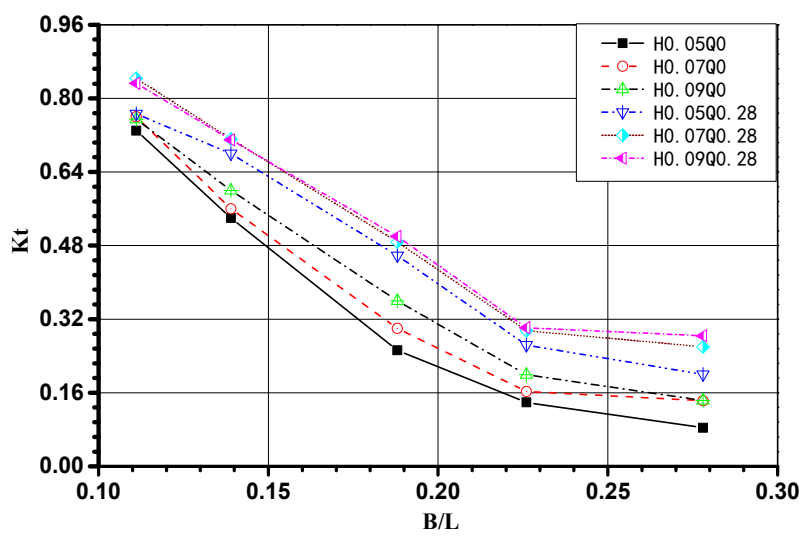

Figure 6 Variation of transmission coefficient with relative width

From the figure 6, it can be seen that the transmission coefficient of the new comb-type FBW decreases with the increase of the relative width, and finally remains constant. When the $\mathrm{B} / \mathrm{L}$ is between 0.225 and 0.28 , the wave length is short and the wave height is effectively reduced. When the groove depth is the same, the transmission coefficient of the FBW increases with the increase of wave height. At the same time, it is not difficult to find that the wave dissipation of $0 \mathrm{~m}$ groove depth (rectangular box-type FBW) is better than $0.28 \mathrm{~m}$ groove depth, but the latter can effectively restrain the wave reflection.

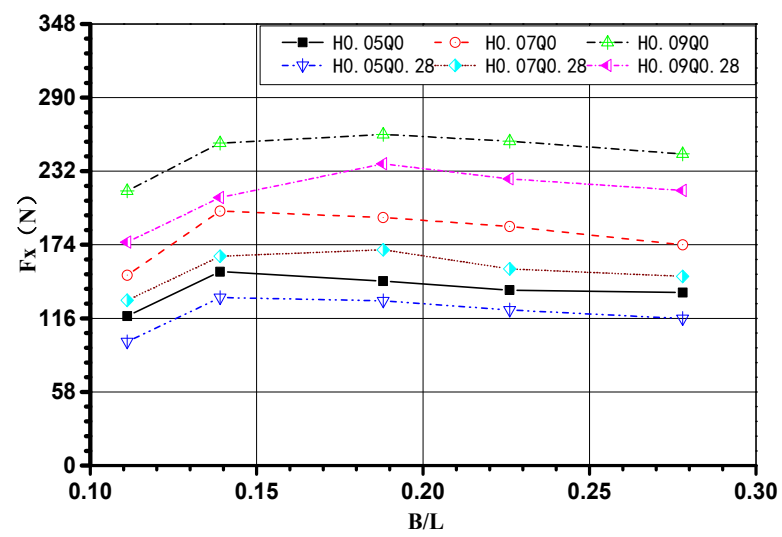

Figure 7 Variation of horizontal wave force with relative width 


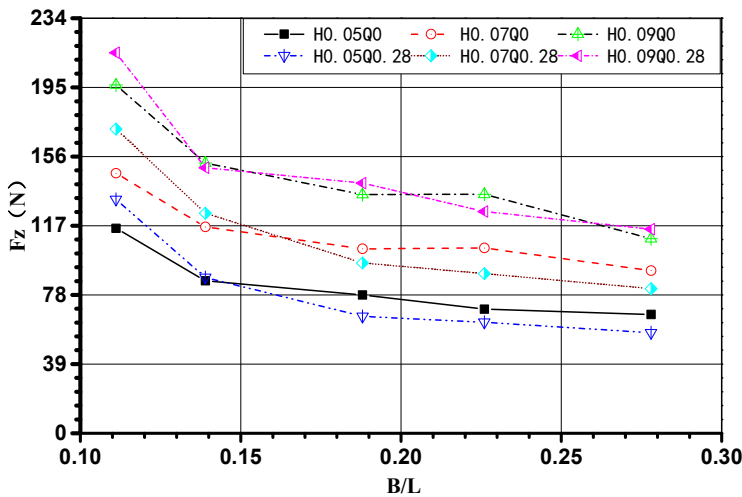

Figure 8 Variation of vertical wave force with relative width

Figure 8 shows that the horizontal wave force acting on the FBW with $0.28 \mathrm{~m}$ groove depth is less than 0 groove depth. It indicates that the existence of the groove depth can reduce the horizontal wave force. However, the existence of groove depth has little effect on vertical wave forces. It is also found that the horizontal wave force increases first and then decreases with the increase of relative width. When the relative width is 0.139 to 0.188 , the transmission coefficient of the new comb-type FBW decreases rapidly with the increase of the relative width, but the horizontal and vertical wave forces change slowly. It is suggested that the relative width should be set in this range as far as possible when designing the fixed FBW. We can also find that the horizontal wave force and vertical wave force are directly proportional to wave height. From the above analyses, effect of groove depth and wave height on hydrodynamic characteristics of the new comb-type FBW are studied, which would provide a theoretical basis for practical application.

\subsection{Effect of relative width and groove depth on hydrodynamic characteristics of the new comb- type FBW}

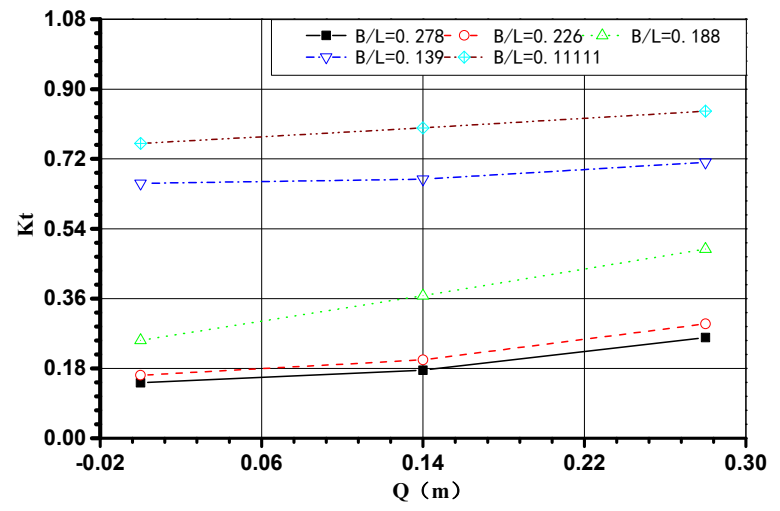

Figure 9 Effect of relative width and groove depth on the transmission coefficient, $\mathrm{Q}$ is the abbreviation of groove depth $(\mathrm{H}=0.07 \mathrm{~m})$

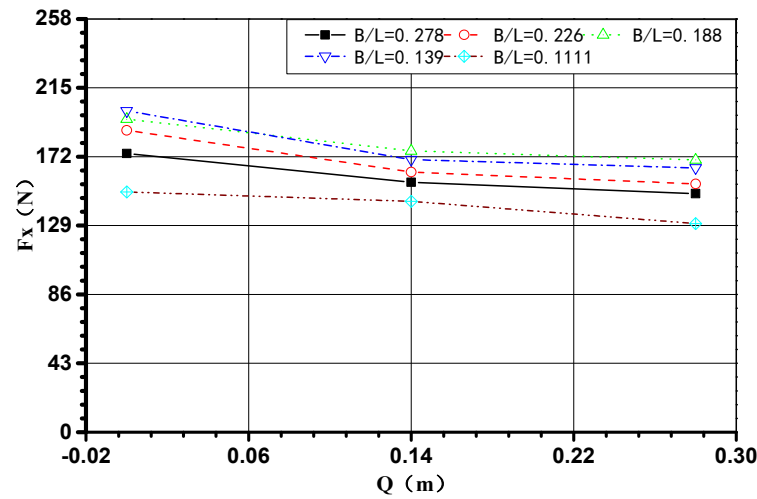

Figure 10 Effect of relative width and groove depth on the horizontal wave force $(\mathrm{H}=0.07 \mathrm{~m})$

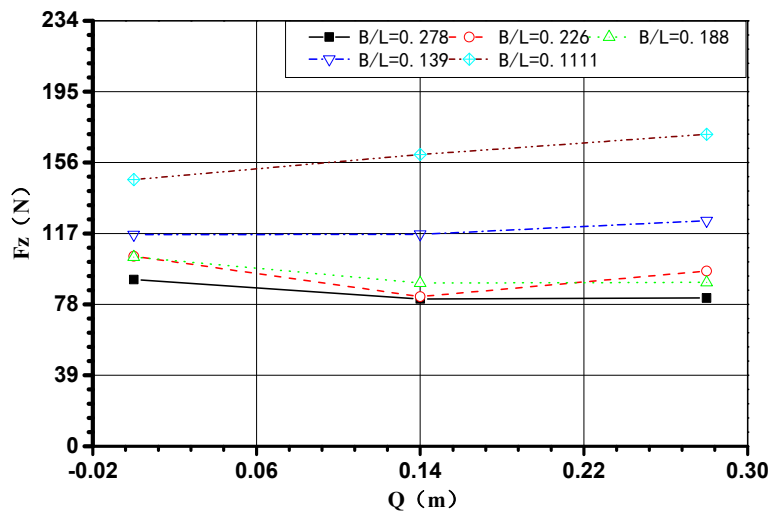

Figure 11 Effect of relative width and groove depth on the vertical wave force $(\mathrm{H}=0.07 \mathrm{~m})$

Under the action of wave height of $0.07 \mathrm{~m}$, the experimental results of the transmission coefficient, horizontal wave force and vertical wave force are shown in Figures 9,10 and 11. As can be seen from the Figure 9, the relative width has a great influence on the transmission coefficient of the FBW, but the groove depth has little influence on it. And the influence of relative width on transmission coefficient of FBW is greater than the horizontal wave force and the vertical wave force. It is also found that the above three hydrodynamic parameters are most affected by small periodic waves, and the large periodic waves have little influence on them. It is not difficult to find from Figure 9 and 10 that the transmission coefficient increases with the increase of groove depth, the horizontal wave force decreases with the increase of groove depth.

\section{Numerical simulation}

We used commercial Computational Fluid Dynamics (CFD) software Flow-3D to analyze wave and FBW interaction and compute wave loads on it. Flow-3D is capable of solving the full 3D Navier-Stokes equations using the volume of fluid (VOF) method. The VOF method enables modeling of the free surface and liquid/gas interface. We first validate the capability of Flow-3D to model regular wave waves. Then we investigate the effects of FBW's elevation on distribution 
of flow field in flume.

\subsection{Validation of Flow-3D for modeling of regular waves}

The computational domain is $1 \mathrm{~m}$ high and $12 \mathrm{~m}$ long. Water depth is set at $0.74 \mathrm{~m}$. The domain is discretized into $120,000 \quad 0.02 \times 0.02 \mathrm{~m}^{2}$ grids in the $\mathrm{x}$-z plane. The water is seawater at $20{ }^{\circ} \mathrm{C}$, and the density is 1.027 $\mathrm{kg} / \mathrm{m} 3$. Regular waves with $0.07 \mathrm{~m}$ wave height and 1.1 $\mathrm{s}$ period are defined as inflow boundary condition at one end. The other end is defined as outflow boundary. Thus the boundary conditions applied are: Xmin-wave, Xmax- outflow, Ymin and Ymax-wall, Zmin-wall and Zmax- specified pressure. An additional wave absorbing component is added before outflow boundary to reduce wave reflections from the boundary and to maintain fluid volume in the domain. The geometry file is imported in STL format and the rendered output is checked to identify whether the grid size is sufficient to maintain geometric shape properly. The fixed FBW is treated as a non-moving object. In order to verify the accuracy of numerical simulation, we lay aside two probes in the flume. The probe1 is located at Xmin, and the probe2 is located at a wavelength distance from probel. The results obtained by numerical simulation are shown in Figure 12. The time domain curves measured by the two probes show that the waveform of the probel is similar with the probe2, except for the difference between the front two waves. It is made sure that the simulation generates the desired wave without dissipation of energy, which confirms the accuracy of the commercial CFD software Flow-3D for modeling of regular waves. Once the right wave is generated, then the fixed FBW simulation is conducted.

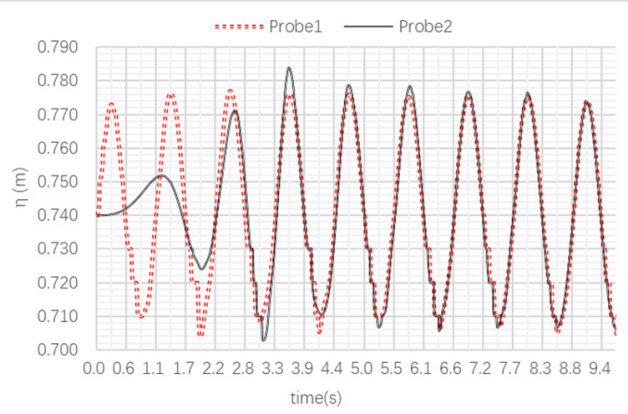

Figure 12 Comparison of the waveform between probel and probe2

\subsection{Flow field around fixed FBW}

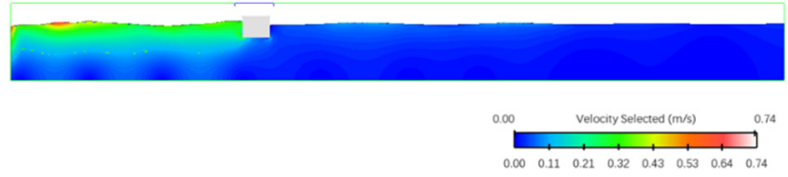

Figure 13 Wave profile and velocity contours

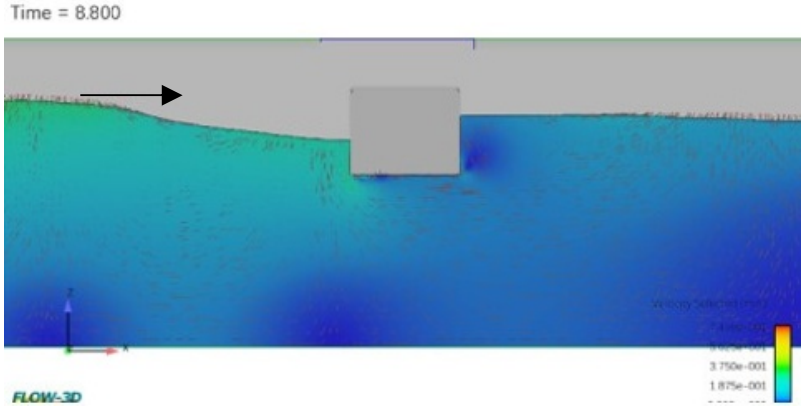

Figure 14 Vectors of water particle velocity (time $=8.8 \mathrm{~s}$ ) As can be seen from Figure 13, since the energy generated by waves is mainly concentrated on the water surface, the height of the FBW fixed on the water surface can block most of the energy in the flow field. As can be seen from Figure 14, the distribution of flow field around the FBW calculated by CFD software Flow-3D is consistent with the experimental results, vortex is found behind the FBW.

This proves that the numerical simulation software Flow-3D has made up for the defects that the flow field is not convenient to be observed in the experiment.

\section{Conclusion}

In this chapter, experimental and numerical investigations are conducted for the transmission coefficient and wave force of a new type of comb-type FBW.

The main conclusions of this paper are as follows:

The relative width has a great influence on the transmission coefficient of the FBW, but the groove depth has little influence on it. And the influence of relative width on transmission coefficient of FBW is greater than that on horizontal wave force and vertical wave force. The above three hydrodynamic parameters are most affected by small periodic waves, and the large periodic waves have little influence on them. The experimental results show that the relative width is 0.139 to 0.188 , the transmission coefficient of the new combtype FBW decreases rapidly with the increase of the relative width, but the horizontal and vertical wave forces change slowly. It is suggested that the relative width should be set in this range as far as possible when designing the fixed FBW.

Numerical investigations of selected experiment cases are conducted using RANS based commercial CFD code Flow3D. The numerical results show a good ability to capture the hydrodynamic interaction effect of a fixed FBW. The velocity changes behand the FBW are also well captured by the numerical simulations. The vortex can be captured with flow modeling software and this points out the direction for further research in the next step.

\section{References}

1. N. Wang, B. Zhao, C.X. Yang, Essential characteristics and evolution law of port generation. Harb. Eng., 2 (2010) 
2. A. Abdolali, M. Kolahdoozan, M. J. Alizadeh, The hydraulic performance of comb-type vertical breakwater. Journal of Hydrodynamics, 3 (2014)

3. Y.C. Li, Z.C. Sun, S.Q. Xu, Hydraulic characteristics of comb caisson breakwater. Journal of Hydrodynamics, 17 ( 2002)

4. C. Li, Experimental study on Floating Breakwaters (master's thesis). Shanghai Jiao Tong University, China. (2008)

5. Z.L. Zou, D.G. Wang, G.W. Li, Experimental study on non-linear wave forces acting on floating breakwaters. The. Ocean. Eng., 21 (2003)

6. Z.L. Zou, Water wave theory and its application. Science Press. Beijing, China. (2005)

7. E. Koutandos, P. Prinos, X. Gironella, Journal of Hydraulic Research, 43 (2005)

8. Y.Z. Cao, Study on hydrodynamic characteristics of non-permeable comb caisson breakwater (master's thesis). Dalian University of Technology, China. (2009)

9. Z. Fang, Study on hydrodynamic characteristics of comb breakwater. (Doctoral dissertation) Dalian University of Technology, China. (2011)

10. Z. Fang, N.C. Zhang, Z.P. Zang, Journal of Waterway and Harbor, 32 (2011)

11. T.M. Sayeed, B. Colbourne, D. Molyneux, A. Akinturk, Experimental and numerical investigation of wave forces on partially submerged bodies in close proximity to a fixed structure. Ocean. Eng., 132 (2017)

12. J. Jin, B. Meng, Computation of wave loads on the superstructures of coastal highway bridges. Ocean. Eng., 38 (2011) 